\title{
Human airway organoids model SARS-CoV-2 high infectiousness and evasion of interferon response
}

Jie Zhou

University of Hong Kong https://orcid.org/0000-0002-2948-3873

Man Chun Chiu

The University of Hong Kong https://orcid.org/0000-0001-9503-6396

\section{Cun LI}

The University of Hong Kong

Xiaojuan Liu

The University of Hong Kong

Xiaoyu Zhao

The University of Hong Kong

Dong Wang

The University of Hong Kong

Yuxuan Wei

The University of Hong Kong

Hin Chu

The University of Hong Kong

Jian-Piao Cai

The University of Hong Kong

Cyril Chik-Yan Yip

The University of Hong Kong

\section{Vincent Poon}

The University of Hong Kong

\section{Ivy Chan}

The University of Hong Kong

\section{Kenneth Kak-Yuen Wong}

The University of Hong Kong

\section{Jasper Fuk-Woo Chan}

The University of Hong Kong

\section{Zhiwei Chen}

AIDS Institute and Department of Microbiology, State Key Laboratory of Emerging Infectious Diseases,

Li Ka Shing Faculty of Medicine, The University of Hong Kong https://orcid.org/0000-0002-4511-2888 Honglin Chen

The University of Hong Kong https://orcid.org/0000-0001-5108-8338 


\section{Hans Clevers}

Hubrecht Institute for Developmental Biology and Stem Cell Research https://orcid.org/0000-00023077-5582

\section{Kwok-Yung Yuen ( $\nabla$ kyyuen@hku.hk)}

University of Hong Kong https://orcid.org/0000-0002-2083-1552

\section{Research Article}

Keywords: Human airway organoids, SARS-CoV-2 infection, interferon response

Posted Date: September 9th, 2020

DOI: https://doi.org/10.21203/rs.3.rs-67556/v1

License: (c) (i) This work is licensed under a Creative Commons Attribution 4.0 International License.

Read Full License 


\section{Human airway organoids model SARS-CoV-2 high infectiousness and evasion of interferon response}

Jie Zhou ${ }^{1,2, \#}$, Man Chun Chiu ${ }^{2, \#}$, Cun $\mathrm{Li}^{2, \#}$, Xiaojuan $\mathrm{Liu}^{2}$, Xiaoyu Zhao ${ }^{2}$, Dong Wang 2 , Yuxuan $\mathrm{Wei}^{2}$, Hin Chu ${ }^{1,2}$, Jian-Piao Cai ${ }^{2}$, Cyril Chik-Yan Yip ${ }^{2}$, Vincent Poon ${ }^{2}$, Ivy Hau-Yee Chan ${ }^{3}$, Kenneth Kak-Yuen Wong ${ }^{3}$, Jasper Fuk-Woo Chan ${ }^{1,2,5}$, Zhiwei Chen ${ }^{1,2,4,5}$ Honglin Chen $^{1,2}$, Hans Clevers $^{6 *}$, Kwok Yung Yuen ${ }^{1,2,5 *}$

${ }^{1}$ State Key Laboratory of Emerging Infectious Diseases, The University of Hong Kong, Hong Kong, China.

${ }^{2}$ Department of Microbiology, Li Ka Shing Faculty of Medicine, The University of Hong Kong, Pokfulam, Hong Kong, China.

${ }^{3}$ Department of Surgery, Li Ka Shing Faculty of Medicine, The University of Hong Kong, Hong Kong, China

${ }^{4}$ AIDS Institute, Li Ka Shing Faculty of Medicine, The University of Hong Kong, Hong Kong, China.

${ }^{5}$ Carol Yu Centre for Infection, The University of Hong Kong, Pokfulam, Hong Kong, China.

${ }^{6}$ Oncode Institute, Hubrecht Institute, Royal Netherlands Academy of Arts and Sciences (KNAW) and University Medical Center (UMC) Utrecht, Utrecht, the Netherlands.

\# These authors contributed equally.

*Correspondence to: Hans Clevers, Email: h.clevers@hubrecht.eu Hubrecht Institute, Utrecht, the Netherlands; and Kwok-Yung Yuen, Email: kyyuen@hku.hk Department of Microbiology, The University of Hong Kong, 102 Pokfulam Road, Pokfulam, Hong Kong Special Administrative Region. 


\section{Summary}

SARS-CoV-2 is more infectious and transmissible in humans than SARS-CoV, despite the genetic relatedness and sharing the same cellular receptor. We sought to assess whether human airway organoids can model SARS-CoV-2 infection in the human airway and elucidate the cellular basis underlying its higher transmissibility. We demonstrate that SARS-CoV-2 can establish a productive infection in human airway organoids, in which ciliated cell and basal cell are infected. Wildtype SARS-CoV-2 carrying a furin cleavage motif exhibits comparable replication kinetics to a mutant virus without the motif. Human airway organoids sustain higher replication of SARSCoV-2 than SARS-CoV, whereas interferon response is more potently induced in the latter than the former. Overall, human airway organoids can model SARS-CoV-2 infection and recapitulate the disposable role of furin cleavage motif for virus transmission in humans. SARS-CoV-2 stealth growth and evasion of interferon response may underlie pre-symptomatic virus shedding in COVID-19 patients, leading to its high infectiousness and transmissibility.

The current pandemic of coronavirus disease 2019 (COVID-19) caused by severe acute respiratory syndrome coronavirus-2 (SARS-CoV-2) has progressed into a public health and socioeconomic crisis globally. Up to date, the total count of confirmed patients has surpassed 24 million with over 800 thousand deaths. COVID-19 is primarily a respiratory illness with a broad spectrum of clinical manifestations, spanning from mild or asymptomatic upper respiratory infection to severe pneumonia or acute respiratory distress syndrome leading to death. ${ }^{1}$ Coronaviruses possess a large positive-sense RNA genome of approximately 30 kilobases. The interaction of viral spike protein and a cognate cellular receptor is the primary determinant for the infectivity to a host and tissue tropism within the host. Upon receptor binding, proteolytic cleavage of spike protein by host serine proteases such as TMPRSS2, cathepsin and furin is required to release the viral genome and initiate replication. Viral RNA synthesis produces both viral genomic RNA and a set of nested subgenomic RNAs. ${ }^{2}$ The latter serves as mRNAs which express structural (spike, envelope, membrane, and nucleocapsid) proteins and accessory proteins. Within the endoplasmic reticulumGolgi intermediate compartment, viral genomes encapsidated by nucleocapsid protein interact with structural proteins to form nascent virions. ${ }^{2}$

In the early outbreak of COVID-19, Zhu et al reported the isolation of SARS-CoV-2 using human primary airway epithelial cells, which was more effective than using Vero E6 cells. ${ }^{3}$ Vero 
E6 or Vero cells are commonly used for virus isolation due to the deficient interferon signaling that favors viral growth. Despite the intact interferon signaling pathway in primary airway epithelial cells, efficient virus isolation using these primary cells is readily anticipated since these airway epithelial cells are the native target cells of SARS-CoV-2. In fact, other human coronaviruses such as hCoV-OC43 and hCoV-NL63, which are refractory to grow in conventional cell lines, have been propagated on human primary airway epithelial cells. ${ }^{4}$ The human airway, from the nasal cavity to terminal bronchiole, is covered with airway epithelium consisting of four major cell types including ciliated cell, goblet cell, club cell and basal cell. In this context, it is implausible that routinely-maintained cell lines, which are composed of homogenous clonalexpansion cells, can simulate the multi-cellular composition and complicated functionality of the human airway epithelium. In order to recapitulate the interaction of respiratory viruses with host cells, scientists utilized primary airway epithelial cells or switched to the primary cells to verify the findings from cell lines. ${ }^{5}$ However, a major obstacle of primary cell culture is the limited proliferation capacity in vitro, the well-recognized Hayflick limit. ${ }^{6}$ As a result, primary cells are unable to be long-term expanded. In addition, regardless of the variation among tissues from different donors, it normally takes at least $4 \sim 6$ weeks to prepare well-differentiated airway epithelial cells. ${ }^{5}$ These issues collectively constrain the application of primary cells for routine experimental purposes. Currently, the morbidity and mortality of COVID-19 pandemic have reached an epic magnitude. However, there is no readily-available and physiological-active in vitro system, superior to human primary bronchial epithelial cells, to model SARS-CoV-2 infection in the human airway, the primary infection site of SARS-CoV-2.

The generation of organoids in vitro represents a major breakthrough in human biology in the past decade. The adult stem cell derived organoid culture has been established for most human organs, including human lung organoid. ${ }^{7,8}$ Upon induction of differentiation for 12 14 days, the long-term expandable, undifferentiated human lung organoids become differentiated airway organoids that faithfully simulate the multi-cellular composition and functional complexity of human airway epithelium, the epithelium lining the human respiratory tract from nasal cavity to bronchiole. As a proof-of-principle, we demonstrated that human airway organoids can discriminate highly infective viruses from less infective ones, thus can be utilized to predict the infectivity of emerging respiratory viruses to humans. ${ }^{7}$ 
SARS-CoV-2 is phylogenetically related to SARS-CoV with $79-82 \%$ sequence similarity. ${ }^{9}$ In 2002 2003, SARS-CoV emerged in China and spread globally. Most SARS patients present as atypical pneumonia with shortness of breath, cough and high fever. SARS-CoV infected more than 8000 individuals with a case-fatality rate of approximately $10 \% .{ }^{10}$ Apart from genetic relatedness, SARS-CoV-2 shares with SARS-CoV the same human cell receptor, angiotensin-converting enzyme 2 (ACE2). However, based on the current epidemiological data, SARS-CoV-2 is more infectious and transmissible than SARS-CoV, yet exhibiting a lower case-fatality rate. The fundamental pathology in human respiratory cells underlying the distinct pathogenicity of two viruses is incompletely understood. In this study, we sought to leverage human airway organoids to model SARS-CoV-2 infection in the human airway. In addition, we studied viral growth of SARS-CoV-2 and cellular response in comparison of SARS-CoV in human airway organoids, a physiological-active model of human respiratory epithelium, aiming to unravel the cellular basis of the high infectiousness of SARS-CoV-2.

We first assessed whether human airway organoids can model SARS-CoV-2 respiratory infection by examining the infectivity and viral growth of SARS-CoV-2 in three lines of human airway organoids derived from primary lung tissues of three donors. Notably, SARS-CoV-2 productively infect all three lines of airway organoids, the viral load increased over $3 \log$ units at 72 hours post inoculation (hpi). The titer of infectious virus was significantly elevated up to $4 \log$ units (Fig. 1a). The presence of viral subgenomic mRNA indicates active viral replication. ${ }^{11} \mathrm{We}$ observed an increasing amount of subgenomic mRNA in SARS-CoV-2 infected airway organoids (Supplementary Fig.1a), indicating active viral replication. In addition, airway organoids underwent progressive cytopathic effect overtime after SARS-CoV-2 inoculation (Supplementary Fig.1b). We further verified productive SARS-CoV-2 infection by immunostaining of viral nucleocapsid protein (NP). Individual infected cells were discernible at 8 hpi. At 24 hpi, virus spread to neighboring cells (Fig. 1b). We proceeded to evaluate cellular tropism of SARS-CoV-2 in human airway organoids. At 24 hours after inoculation, we performed confocal imaging of the infected airway organoids after co-staining of viral NP and FOXJ1 or P63, the marker of airway ciliated and basal cell respectively. As shown in Fig. 1c, both ciliated cells and basal cells were infected by SARS-CoV-2.

We also performed transmission electron microscopy to examine human airway organoids and SARS-CoV-2-infected organoids. Many cells in airway organoids possess the cilium structure 
with microtubules of $9+2$ arrangement, while others show the ultrastructure of goblet cell with secretory vesicles in the apical cytoplasm (Fig. 1d and Fig.1e), indicating an appreciable mucociliary differentiation of airway organoids as we demonstrated previously. ${ }^{7}$ Virion particles with an average size of 70 80 nm and the typical "corona" projections are readily discernible within the cells of inoculated airway organoids (Fig. 1f and Fig.1g).

As aforementioned, cellular serine proteases prime coronaviruses for efficient entry. Recent studies have demonstrated that serine protease TMPRSS2 enhances SARS-CoV-2 cellular entry and replication capacity. ${ }^{12}$ We measured the expression levels of ACE2 and pro-viral proteases in human airway organoids in comparison to undifferentiated lung organoids from which airway organoids are derived. Compared to the undifferentiated lung organoids (LO), human airway organoids (AO) exhibited significantly upregulated ACE2 and all pro-viral proteases (Fig. 2a). ACE2 is clearly shown on the apical membrane in the cells of airway organoids (Fig. 2b). Collectively, human airway organoids faithfully simulate native airway epithelium, and exhibit high susceptibility to SARS-CoV-2.

One of the notable features of SARS-CoV-2 is the presence of a polybasic furin cleavage PRRA motif in S1/S2 boundary in spike protein, ${ }^{13}$ which is absent in the closely-related bat coronaviruses. $^{14}$ In avian influenza viruses of H5 and H7 hemagglutinin (HA) subtypes, acquisition of the polybasic cleavage site in the envelope protein HA is associated with heightened pathogenicity. ${ }^{15}$ Hoffmann et al demonstrated that the PRRA motif was essential for SARS-CoV2 cellular entry in Calu-3 cells. ${ }^{16}$ Jaimes et al documented that the PRRA insertion allows furin cleavage of SARS-CoV-2 spike protein and may enhance spike protein cleavage by other proteases. ${ }^{17}$ However, a recent study suggested the furin cleavage motif is not essential for the fusion activity of SARS-CoV-2 spike protein in the environment where serine proteases are present, such as the human airway. ${ }^{18}$ Given the controversies, we sought to evaluate the role of the PRRA motif for SARS-CoV-2 replication in human airway organoids. We obtained a SARS-CoV2 variant with PRRA motif deletion through plaque purification and compared the replication kinetics of wildtype and deletion mutant virus. Interestingly, the deletion mutant virus (Del) and wildtype virus (WT) replicated to a similar titer in human airway organoids (Fig. 3a). We recently reported SARS-CoV-2 infection in human intestinal organoids. ${ }^{19}$ Indistinguishable replication of wildtype and deletion mutant SARS-CoV-2 was also manifested in human small intestinal 
organoids (Fig. 3b). Thus, the results demonstrated the furin cleavage is dispensable for SARSCoV-2 propagation in human airway and intestinal epithelial cells.

Ogando et al reported rapid acquisition of the deletion mutation and strong selection of the deletion mutant virus when SARS-CoV-2 was propagated in Vero E6 cells. ${ }^{20}$ We have recently developed a duplex digital PCR assay to quantify PRRA motif variants in SARS-CoV-2 samples. ${ }^{21}$ Herein, we examined the abundance of both variants in the culture media from airway organoids infected with plaque-purified wildtype or deletion mutant virus. Wildtype and deletion mutant variants were exclusively detected from airway organoids infected with the respective virus (Supplementary Fig.2). We did not observe the appearance of deletion mutant variant when plaque-purified wildtype virus replicated in human airway organoids for 72 hours. Therefore, viral genomic region of the $\mathrm{S} 1 / \mathrm{S} 2$ cleavage site remains stable during $\mathrm{SARS}-\mathrm{CoV}-2$ replication in human airway organoids.

Based on the clinical manifestations and epidemiological findings, SARS-CoV-2 is apparently more infectious than SARS-CoV. A possible explanation is that SARS-CoV-2 per se replicates more robustly than SARS-CoV in human respiratory epithelial cells. Alternatively, more severe disease manifestation of SARS than COVID-19 enables readily identification of SARS patients, and subsequent interventions prevent human-to-human transmission of SARS-CoV. As a result, SARS-CoV appears to be less infectious than SARS-CoV-2. To address which is the authentic scenario or whether both are operational, we compared the replication capacity of SARSCoV and SARS-CoV-2 in human airway organoids. As shown in Fig. 4a, viral load in the culture medium of SARS-CoV-2-infected organoids, based on the detection of copy number of viral RdRp gene, was around 10-fold higher than that in SARS-CoV-infected organoids. To verify the result, we detected the copy number of another viral gene, nucleocapsid (NP). The same trend was observed, significantly more NP RNA molecules were generated by SARS-CoV-2 than SARS$\mathrm{CoV}$ in human airway organoids (Supplementary Fig.3). Intriguingly, TCID 50 assay showed indistinguishable viral titers in Vero E6 cells using culture media collected from SARS-CoV-2and SARS-CoV-infected organoids.

Viral gene copy number in the samples reveals the abundance of total RNA species of RdRp and NP gene, including viral genomic RNA and subgenomic RNA. To quantify the copy numbers of viral RNAs, we used two pairs of primers targeting the conserved region in RdRp or NP genes of two viruses and virus-specific probes, which ensures an equal amplification of RNA 
molecules of two viruses and high specificity of the assay simultaneously. Thus, the assay enables accurate measurement and direct comparison of the amount of viral RNAs generated from the two viruses. The inconsistent pattern of viral gene copy number and viral titer could be attributed to Vero E6 cells used to measure viral titer. Ogando et al reported that SARS-CoV replicated more efficiently than SARS-CoV-2 in Vero E6, ${ }^{20}$ the cells used for our TCID 50 assay. Thus, TCID 50 assay itself is a biased measurement for comparing titers of the two viruses, since Vero E6 cells favor the outgrowth of SARS-CoV over SARS-CoV-2. Conceivably, more robust replication of SARS-CoV-2 than SARS-CoV would be masked, instead shown as indistinguishable viral titers when the medium samples of two viruses are titrated in Vero E6 cells. Unfortunately, as far as we know, few cell lines can be used for unbiased titration of these two viruses. We then detected the infection rate of SARS-CoV and SARS-CoV-2 in the infected airway organoids by flow cytometry. At 24 hpi, the percentage of virus-infected cells was around 3-fold higher in SARSCoV-2-infected organoids than SARS-CoV-infected organoids (Fig. 4b). Therefore, viral load detection and flow cytometry analysis consistently reveal higher infectivity and replication potential of SARS-CoV-2 than SARS-CoV in human airway organoids.

As aforementioned, SARS-CoV produced significantly more infectious virions than SARS-CoV-2 in Vero E6 cells. ${ }^{20}$ The disparate replication capacity of SARS-CoV and SARSCoV-2 in Vero E6 cells and human airway organoid is probably ascribed to cellular interferon response, which is active in human airway organoids, albeit defective in Vero E6 cells. Interferons are key antiviral cytokines produced during innate immune detection of viral infections. Type I IFNs systematically control infection, whilst type III IFNs eliminate infection locally at mucosal surfaces including epithelium lining the respiratory and gastrointestinal tracts. Mouse and human respiratory epithelial cells preferentially secrete type III IFNs in response to influenza virus and respiratory syncytial virus infections. ${ }^{22,23}$ In addition, expression of type III IFN receptor complex is most commonly restricted to the epithelial cells at mucosal surfaces. ${ }^{24}$ Type I and type III IFNs trigger almost identical downstream signals and lead to activation of hundreds of IFN-stimulated genes that coordinate the eradication of pathogens and infected cells, and meanwhile produce a pro-inflammatory response that contributes to inflammation and clinical symptoms of viral infections.

We then examined the expression levels of a panel of IFN genes in airway organoids infected by SARS-CoV and SARS-CoV-2 (Fig. 4c). SARS-CoV significantly stimulated the 
induction of type III IFNs including IFN $\lambda 2$ and IFN $\lambda 3$, type I IFN (IFN $\alpha$ ) and type II IFN (IFN $\gamma$ ). However, IFN $\lambda 3$ and IFN $\gamma$ were only marginally induced in SARS-CoV-2-infected airway organoids; and type I IFNs and IFN 22 were barely stimulated. As a result, the induction of IFN $\lambda 2$, IFN $\alpha$ and IFN $\gamma$ was significantly higher in SARS-CoV infection than SARS-CoV-2 infection. Potent induction of IFN response was shown in human airway organoids infected by influenza A virus of H1N1 pandemic strain (Supplementary Fig. 4). The results suggested that SARS-CoV-2 developed strong antagonism to evade IFN response.

Airway organoids derived from adult stem cells can recapitulate the key features of native airway epithelium to a near-physiological level. In this study, we demonstrate that human airway organoids are highly susceptible to SARS-CoV-2. Currently, it remains a controversial issue regarding the role of furin cleavage motif for the infectivity of SARS-CoV-2 to humans. The conclusion drawn from each in vitro study using cell line appears evidence-based and rational. However, these meticulously-conducted studies reached conflicting conclusions. ${ }^{16,}{ }^{18}$ The inspection in human airway organoids indicates furin cleavage is dispensable for the infectivity and replication capacity of SARS-CoV-2. Furthermore, viral genome region harboring the furin cleavage motif is very stable during SARS-CoV-2 replication in human airway organoids, in contrast to the rapid acquisition of the deletion mutation in Vero E6 cells. Despite contradictory to the findings of some in vitro studies, ${ }^{16,17}$ our observation is actually compatible to the real-life scenarios in human and related animal hosts. The furin cleavage motif is absent in closely-related bat coronavirus RaTG13 and pangolin coronavirus, leading to a speculation that acquisition of the motif may be essential for the cross-species transmission and productive infection of SARS-CoV2 in humans. ${ }^{14}$ Shortly afterwards, a bat coronavirus closely related to SARS-CoV-2 (with 97\% sequence similarity) was identified, and the virus possesses a similar furin cleavage motif in the spike protein. ${ }^{25}$ In addition, we and others found that SARS-CoV-2 without the furin cleavage motif was literally transmissible in humans. ${ }^{21}$ Based on these studies, the furin cleavage motif is not essential for animal-to-human and human-to-human transmissions. Namely, the comparable replication capacity of these two viruses in human airway organoids adequately recapitulates the redundant role of the furin cleavage motif for viral growth of SARS-CoV-2 in humans, which highlights these airway organoids as a physiological-active tool to model SARS-CoV-2 infection in the human airway. 
Furthermore, more robust viral growth of SARS-CoV-2 than SARS-CoV in the airway organoids and evasion of IFN response may recapitulate and underlie the distinct transmission profile of COVID-19 and SARS. In SARS-CoV-2 patients, the highest viral load was detected at the time of symptom onset. ${ }^{26}$ Infectiousness of COVID-19 patients peaked on or before symptom onset. Pre-symptomatic or asymptomatic virus shedding of COVID-19 patients has been documented extensively. ${ }^{1,27}$ In contrast, a prospective study of a large cohort of SARS patients showed that the patients exhibited increasing viral load during the first week that peaked on at day $7 \sim 10$ days after the onset of symptoms. ${ }^{28}$ Human-to-human virus transmission occurred days after illness. Given these observations in COVID-19 and SARS patients, we believe the higher infectiousness of SARS-CoV-2 is attributed to more robust but "stealth" replication of SARSCoV-2 due to the evasion of IFN response in the early phase of infection, which is clinically manifested as virus shedding in pre-symptomatic COVID-19 patients. SARS-CoV-2 evasion of IFN response was also observed in the infected human primary bronchial epithelial cells. ${ }^{29}$ In stark contrast, IFN production, especially IFN $\gamma$ induced in SARS-CoV-infected airway epithelial cells leads to inflammation in respiratory tissues and early appearance of symptoms in SARS patients. IFN $\gamma$-related cytokine storm was induced in SARS patients' sera and contributable to the immunopathological damage of the patients. ${ }^{30}$

Taken together, SARS-CoV-2 evades IFN response and replicates robustly in human airway organoids. SARS-CoV-2 stealth growth and evasion of host antiviral response may underlie the pre-symptomatic virus shedding in COVID-19 patients, leading to its high infectiousness and transmissibility. The human airway organoid model of SARS-CoV-2 respiratory infection, and the intestinal organoid model of SARS-CoV-2 intestinal infection, ${ }^{19}$ can serve as powerful and physiological-active in vitro models to understand human SARS-CoV-2 infection.

Acknowledgments: We thank the Center of PanorOmic Sciences and Electron Microscope Unit, Li Ka Shing Faculty of Medicine, University of Hong Kong, for assistance in confocal imaging flow cytometry, and electron microscopy.

Funding: This work was partly supported by funding from Health and Medical Research Fund (HMRF, 17161272, 19180392) of the Food and Health Bureau of the HKSAR government to J.Z; General Research Fund (GRF, 17105420) of the Research Grants Council of HKSAR government 
275 to J.Z.; Theme-based Research Scheme (T11-707/15-R) of the Research Grants Council, the 276 HKSAR Government to K.Y.Y.; the High Level Hospital-Summit Program in Guangdong, The 277 University of Hong Kong-Shenzhen Hospital to K.Y.Y.; and the donations of the Shaw Foundation 278 Hong Kong, May Tam Mak Mei Yin, Richard Yu and Carol Yu, Michael Seak-Kan Tong, 279 Respiratory Viral Research Foundation Limited, Hui Ming, Hui Hoy \& Chow Sin Lan Charity 280 Fund Limited, and Chan Yin Chuen Memorial Charitable Foundation to K.Y.Y..

281 Author contributions: J.Z., H.Clevers and K.Y.Y. designed and supervised the study. M.C.C., 282 C.L., X.L., X.Z., D.W., Y.W., H.Chu., V.P. and C.C.-Y.Y. performed the experiments. J.-P.C. 283 prepared the NP antibodies. J.Z., M.C.C. C.L., X.L., X.Z., and J.F.-W.C. analyzed the data. I.H.284 Y.C. and K.K.-Y.W. provided human lung tissues. J.Z., H.Clevers, Z.C., H.Chen., and K.Y.Y. 285 wrote the manuscript.

286 Competing interests: The authors declare no conflict of interest. 


\section{References}

1. Chan, J.F. et al. A familial cluster of pneumonia associated with the 2019 novel coronavirus indicating person-to-person transmission: a study of a family cluster. Lancet 395, 514-523 (2020).

2. Fehr, A.R. \& Perlman, S. Coronaviruses: an overview of their replication and pathogenesis. Methods $\mathrm{Mol}$ Biol 1282, 1-23 (2015).

3. Zhu, N. et al. A Novel Coronavirus from Patients with Pneumonia in China, 2019. N Engl J Med 382, $727-$ $733(2020)$.

4. Dijkman, R. et al. Isolation and characterization of current human coronavirus strains in primary human epithelial cell cultures reveal differences in target cell tropism. $J$ Virol 87, 6081-6090 (2013).

5. Matrosovich, M.N., Matrosovich, T.Y., Gray, T., Roberts, N.A. \& Klenk, H.D. Human and avian influenza viruses target different cell types in cultures of human airway epithelium. Proc Natl Acad Sci US A 101, 4620-4624 (2004).

6. Hayflick, L. \& Moorhead, P.S. The serial cultivation of human diploid cell strains. Exp Cell Res 25, 585621 (1961).

7. Zhou, J. et al. Differentiated human airway organoids to assess infectivity of emerging influenza virus. Proc Natl Acad Sci U S A 115, 6822-6827 (2018).

8. Sachs, N. et al. Long-term expanding human airway organoids for disease modeling. EMBO J 38 (2019).

9. Chan, J.F. et al. Genomic characterization of the 2019 novel human-pathogenic coronavirus isolated from a patient with atypical pneumonia after visiting Wuhan. Emerg Microbes Infect 9, 221-236 (2020).

10. Perlman, S. \& Dandekar, A.A. Immunopathogenesis of coronavirus infections: implications for SARS. Nat Rev Immunol 5, 917-927 (2005).

11. Wolfel, R. et al. Virological assessment of hospitalized patients with COVID-2019. Nature 581, 465-469 (2020).

12. Hoffmann, M. et al. SARS-CoV-2 Cell Entry Depends on ACE2 and TMPRSS2 and Is Blocked by a Clinically Proven Protease Inhibitor. Cell 181, 271-280 e278 (2020).

13. Lau, S.Y. et al. Attenuated SARS-CoV-2 variants with deletions at the S1/S2 junction. Emerg Microbes Infect 9, 837-842 (2020).

14. Andersen, K.G., Rambaut, A., Lipkin, W.I., Holmes, E.C. \& Garry, R.F. The proximal origin of SARSCoV-2. Nat Med 26, 450-452 (2020).

15. Nao, N. et al. Genetic Predisposition To Acquire a Polybasic Cleavage Site for Highly Pathogenic Avian Influenza Virus Hemagglutinin. mBio 8 (2017).

16. Hoffmann, M., Kleine-Weber, H. \& Pohlmann, S. A Multibasic Cleavage Site in the Spike Protein of SARS-CoV-2 Is Essential for Infection of Human Lung Cells. Mol Cell 78, 779-784 e775 (2020).

17. Jaimes, J.A., Millet, J.K. \& Whittaker, G.R. Proteolytic Cleavage of the SARS-CoV-2 Spike Protein and the Role of the Novel S1/S2 Site. iScience 23, 101212 (2020).

18. Xia, S. et al. The role of furin cleavage site in SARS-CoV-2 spike protein-mediated membrane fusion in the presence or absence of trypsin. Signal Transduct Target Ther 5, 92 (2020).

19. Zhou, J. et al. Infection of bat and human intestinal organoids by SARS-CoV-2. Nat Med (2020).

20. Ogando, N.S. et al. SARS-coronavirus-2 replication in Vero E6 cells: replication kinetics, rapid adaptation and cytopathology. J Gen Virol (2020).

21. Wong, Y.C. et al. Natural transmission of bat-like SARS-CoV-2PRRA variants in COVID-19 patients. Clin Infect Dis (2020).

22. Iwasaki, A. \& Pillai, P.S. Innate immunity to influenza virus infection. Nat Rev Immunol 14, 315-328 (2014).

23. Villenave, R. et al. Induction and Antagonism of Antiviral Responses in Respiratory Syncytial VirusInfected Pediatric Airway Epithelium. J Virol 89, 12309-12318 (2015).

24. Sommereyns, C., Paul, S., Staeheli, P. \& Michiels, T. IFN-lambda (IFN-lambda) is expressed in a tissuedependent fashion and primarily acts on epithelial cells in vivo. PLoS Pathog 4, e1000017 (2008).

25. Zhou, H. et al. A Novel Bat Coronavirus Closely Related to SARS-CoV-2 Contains Natural Insertions at the S1/S2 Cleavage Site of the Spike Protein. Curr Biol 30, 2196-2203 e2193 (2020).

26. He, X. et al. Temporal dynamics in viral shedding and transmissibility of COVID-19. Nat Med 26, 672-675 (2020).

27. Long, Q.X. et al. Clinical and immunological assessment of asymptomatic SARS-CoV-2 infections. Nat Med (2020). 
28. Peiris, J.S. et al. Clinical progression and viral load in a community outbreak of coronavirus-associated SARS pneumonia: a prospective study. Lancet 361, 1767-1772 (2003).

29. Blanco-Melo, D. et al. Imbalanced Host Response to SARS-CoV-2 Drives Development of COVID-19. Cell 181, 1036-1045 e1039 (2020).

30. Huang, K.J. et al. An interferon-gamma-related cytokine storm in SARS patients. J Med Virol 75, $185-194$ (2005). 
$351 \quad$ Fig. 1

a

o Donor 1

口 Donor 2
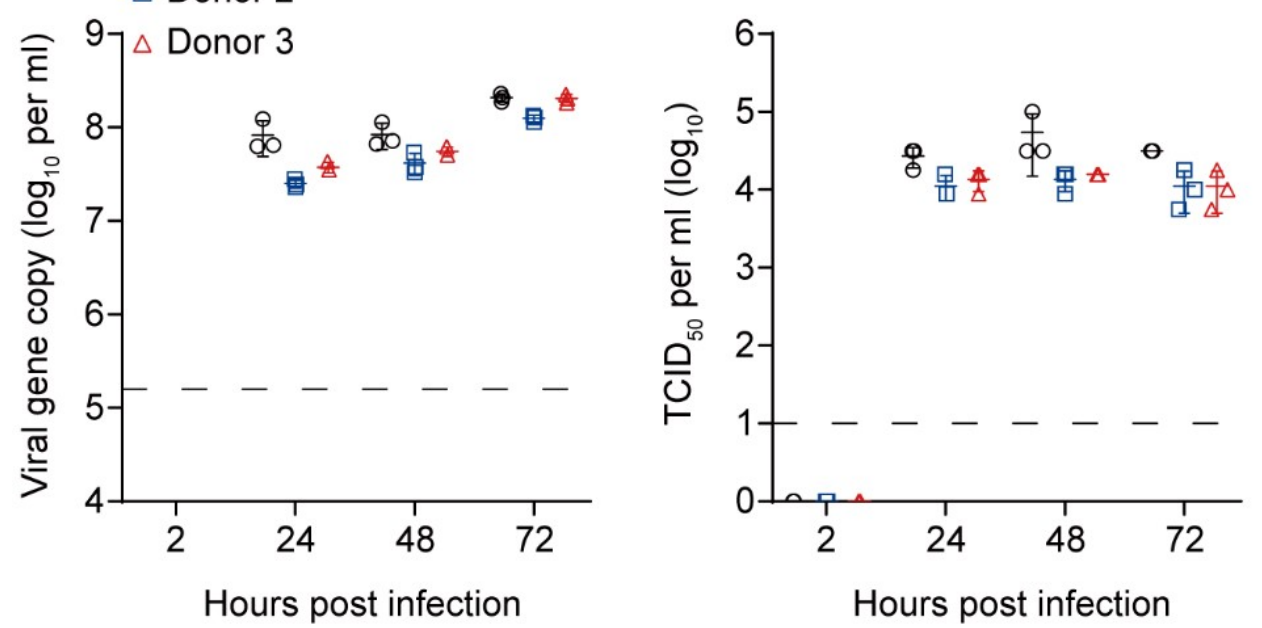

b
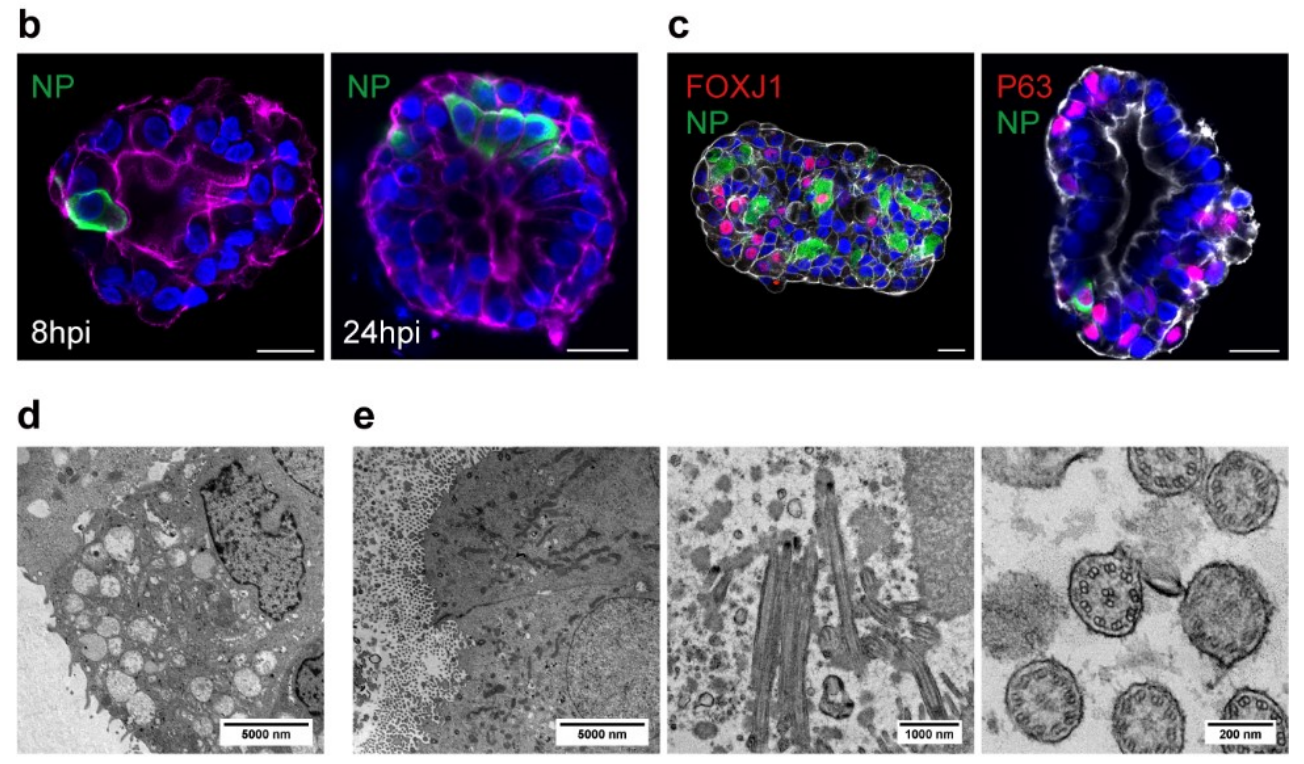

f

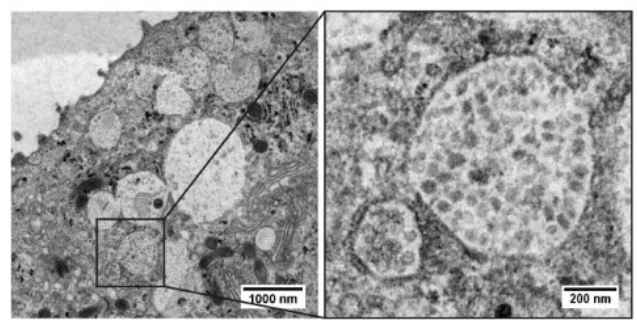

g

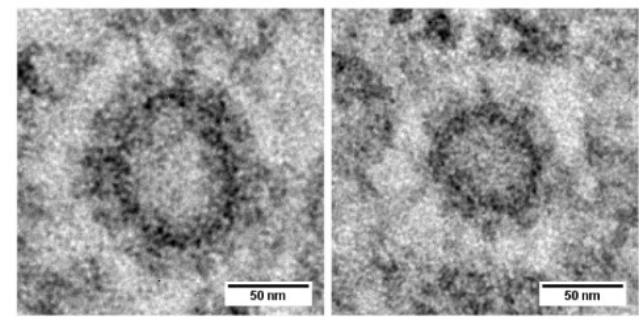


354 Fig. 1. Active replication of SARS-CoV-2 in human airway organoids (HAO). (a) Culture

355 media were harvested from three lines of SARS-CoV-2 infected HAO at the indicated hours post 356 infection and applied to viral load detection and viral titration by TCID $_{50}$ assay. Data represent 357 mean and SD of a representative experiment, $n=3$. Independent experiments were performed three 358 times in triplicate. Dashed line indicates detection limit. (b) SARS-CoV-2-infected HAOs were 359 fixed at 8 or 24 hpi and immuno-stained to identify viral NP (green) positive cells. Nuclei and 360 actin filaments were counterstained with DAPI (blue) and Phalloidin-647 (purple) respectively. 361 Scale bar, $20 \mu \mathrm{m}$. (c) After fixation at $24 \mathrm{hpi}$, SARS-CoV-2-infected HAOs were co-stained with $362 \alpha-\mathrm{NP}$ (green) and $\alpha-\mathrm{FOXJ} 1$ (red, left) or $\alpha-\mathrm{P} 63$ (red, right) to examine cell tropism. Nuclei and 363 actin filaments were counterstained with DAPI (blue) and Phalloidin-647 (white) respectively. 364 Scale bar, $20 \mu \mathrm{m}$. (d) Transmission electron microscopy shows ultrastructural morphology of a 365 goblet cell in HAO. (e) Images of transmission electron microscopy illustrate ultrastructural 366 morphology of ciliated cells (left) in HAO, including the longitudinal section (middle) and cross367 section (right) of cilia with microtubules of 9+2 arrangement. (f)Transmission electron microscopy 368 shows the presence of virion particles in a secretory vesicle in SARS-CoV-2-infected cells in 369 HAO; the boxed area is amplified on the right. (g) Images of transmission electron microscopy 370 show the detailed morphology of virion particles in cytoplasm of SARS-CoV-2-infected cells in 371 HAO.

372 

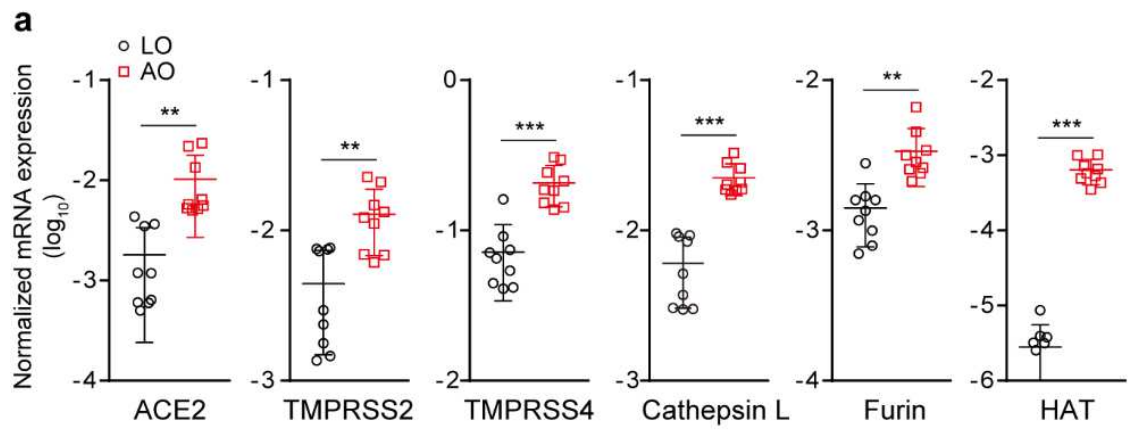

b
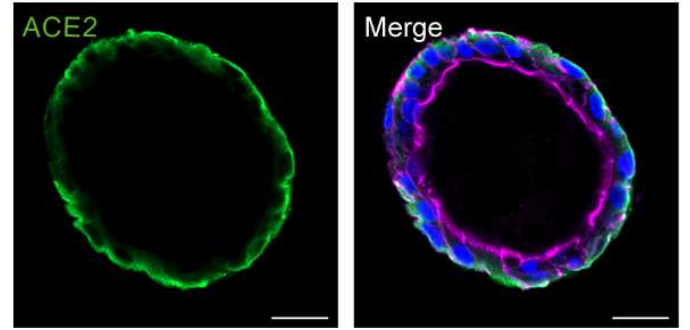

Fig. 2. Expression of pro-viral host factors in human airway organoids. (a) Three lines of 376 undifferentiated human lung organoids (LO) and the differentiated human airway organoids (AO) 377 derived from the former were applied to RT-qPCR assay to detect transcriptional levels of ACE2, 378 TMPRSS2, TMPRSS4, Cathepsin L, Furin and HAT. Data represent mean and SD of three 379 independent experiments, $\mathrm{n}=9$. Two-tailed Student's t test. (b) Differentiated human airway 380 organoids were applied to immunofluorescence staining to label human ACE2 protein (green). 381 Nuclei and actin filaments were counterstained with DAPI (blue) and Phalloidin-647 (purple) 382 respectively. Scale bar, $20 \mu \mathrm{m}$. 

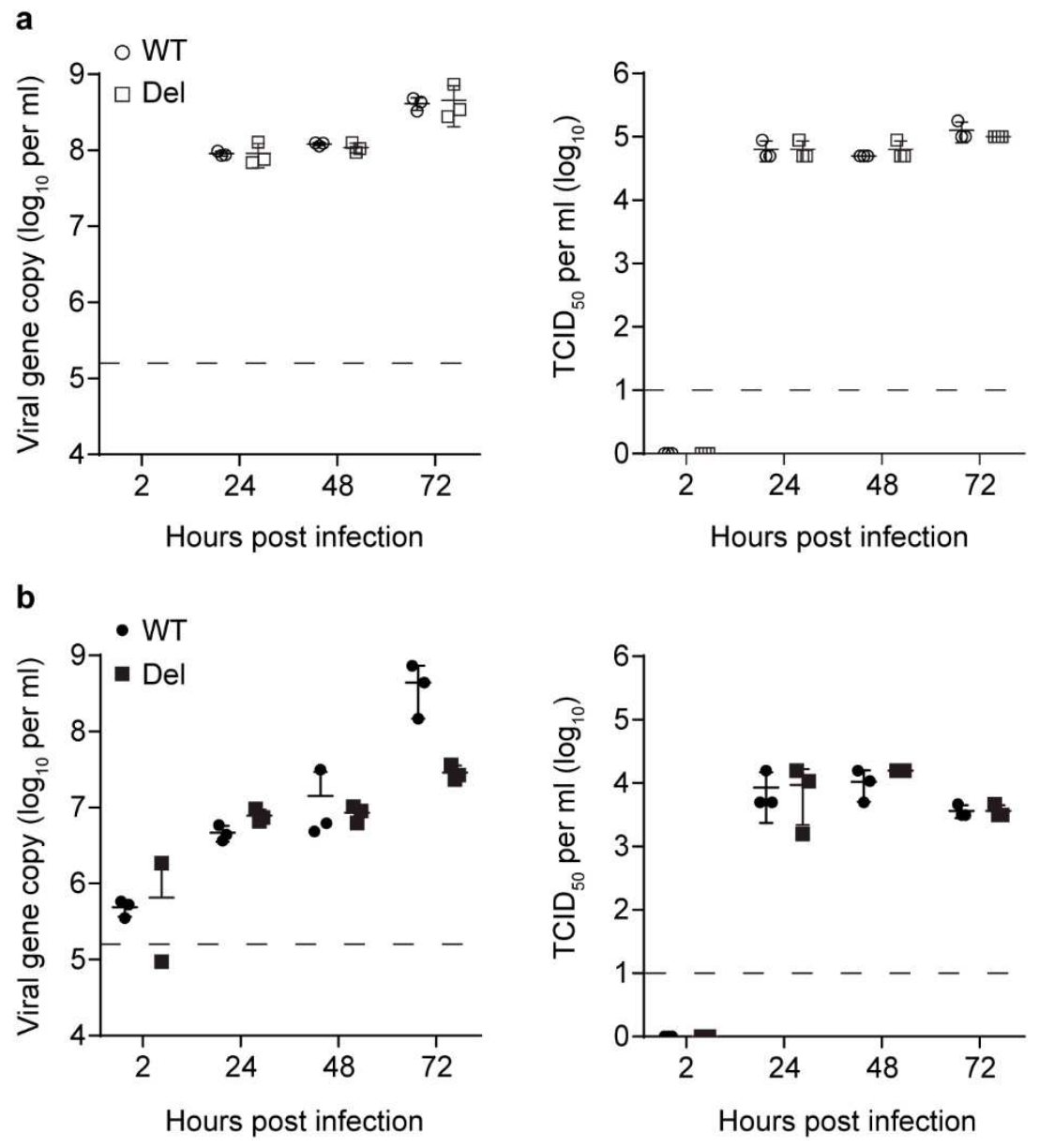

Fig. 3. Comparison of wildtype and deletion mutant SARS-CoV-2 in human airway and intestinal organoids. (a) At the indicated hour post infection of wildtype (WT) or deletion mutant (Del) SARS-CoV-2 in human airway organoids, culture media were harvested and applied to viral load detection and viral titration by $\mathrm{TCID}_{50}$ assay. Data represent mean and SD of a representative experiment, $n=3$. Independent experiments were performed three times in duplicate or triplicate. Dashed line indicates detection limit. (b) Culture media were harvested from SARS-CoV-2 wildtype (WT) or deletion mutant (Del) infected human intestinal organoids and applied to viral load detection and viral titration by $\mathrm{TCID}_{50}$ assay. Data represent mean and SD of a representative experiment, $n=3$. Independent experiments were performed three times in triplicate. Dashed line 
Fig.4

a

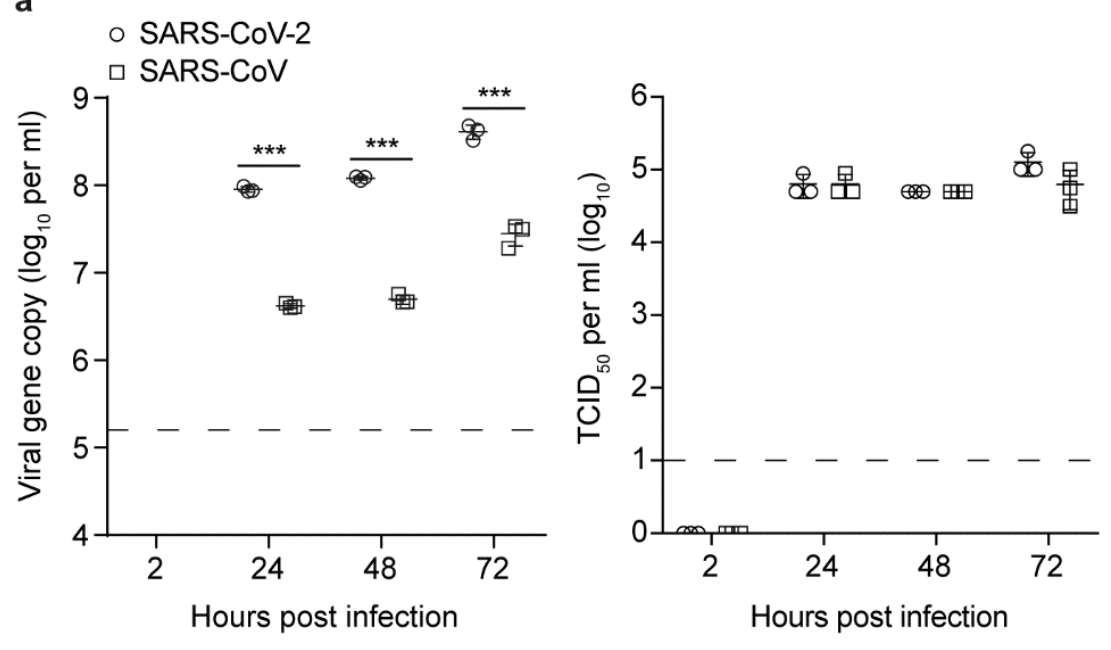

b
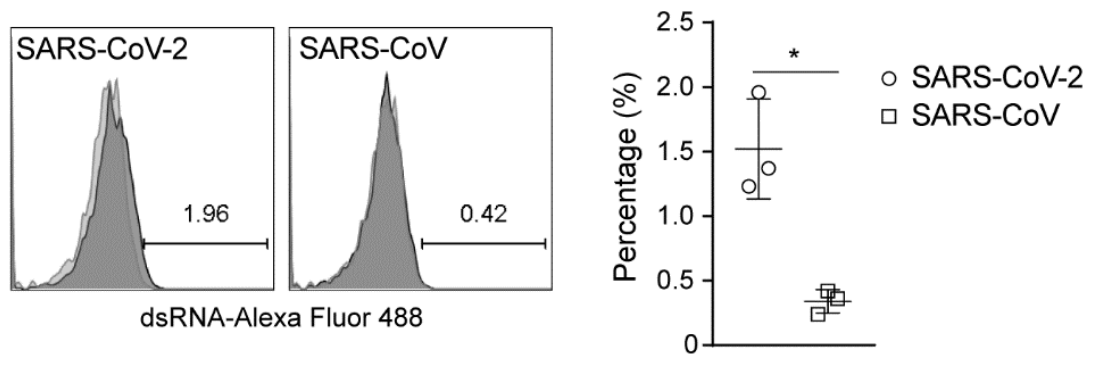

C

c $\mathrm{MOCK}$
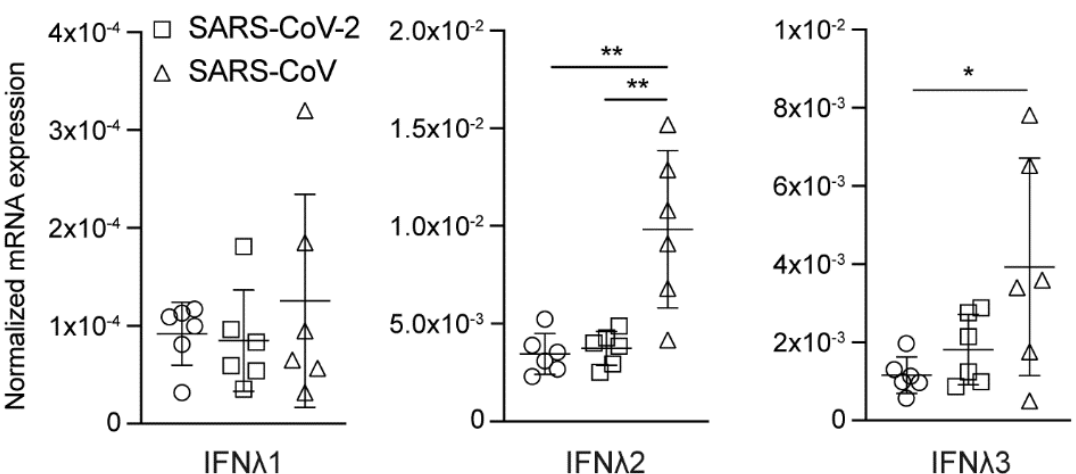

IFN $\lambda 3$

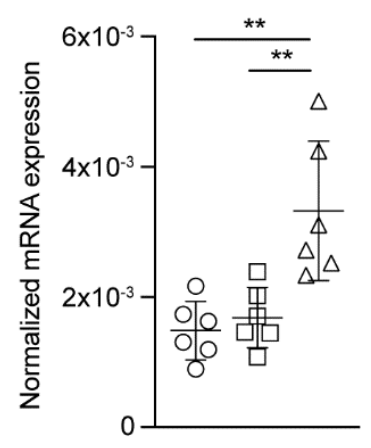

IFNa

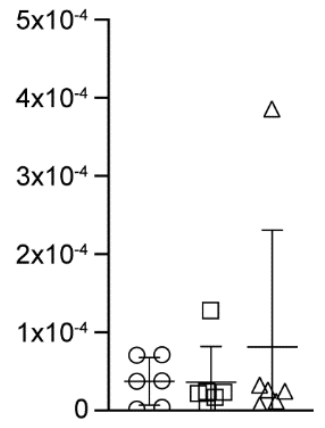

IFN $\beta$

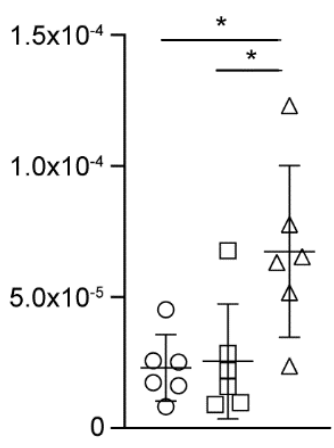

IFNy 
401 Fig.4. Comparison of viral growth of SARS-CoV-2 and SARS-CoV and IFN response in

402 human airway organoids. (a) At the indicated hour post infection of SARS-CoV-2 or SARS-

$403 \mathrm{CoV}$, culture media were harvested from the human airway organoids and applied to viral load 404 detection and viral titration by TCID $_{50}$ assay. Data represent mean and SD of a representative 405 experiment, $n=3$. Independent experiments were performed three times in triplicate. Dashed line 406 indicates detection limit. Two-tailed Student's t test. (b) Representative histograms show the 407 percentage of virus positive cells in human airway organoids infected by SARS-CoV-2 or SARS$408 \mathrm{CoV}$ at 24 hours post infection. Data on the right panel represent mean and SD of a representative 409 experiment, $n=3$. Two-tailed Student's $t$ test. (c) Induction of IFNs in human airway organoids at 41048 hours post infection. Results show GAPDH normalized expression level in the infected human 411 airway organoids and the mock-infected organoids. Data represent mean and SD of two 412 independent experiments, $\mathrm{n}=6$. Two-tailed Student's t test. 


\section{Methods}

Establishment, maintenance and differentiation of human airway and intestinal organoids. After ethics approval by the Institutional Review Board of the University of Hong Kong/Hospital Authority Hong Kong West Cluster (UW13-364), three lines of human lung organoids were previously established using the human lung tissues from patients who underwent surgical resection. ${ }^{7}$ Briefly, human lung organoids are maintained in the expansion medium and passaged every 2-3 weeks. To induce differentiation into airway organoids, undifferentiated lung organoids are transferred into differentiation medium and incubated for 12-14 days. Human intestinal organoids were established using the human intestinal tissues from patients as previously reported. $^{19}$

The undifferentiated lung organoids and differentiated airway organoids were harvested and applied to RNA extraction using MiniBEST Universal RNA Extraction kit (Takara), followed by reverse transcription using Transcriptor First Strand cDNA Synthesis Kit (Roche) and oligo(dT) primer. The resultant cDNAs were used to measure mRNA expression levels of host genes using the LightCycler 480 SYBR Green I Master Mix (Roche). Photomicrographs of the organoids were acquired using Nikon Eclipse TS100 Inverted Routine Microscope. Unless stated otherwise, all examinations and infections were conducted in the differentiated human airway or intestinal organoids.

Virus infection and detection.

SARS-CoV-2 (GenBank accession number MT230908) and SARS-CoV-2 with deletion mutant (GISAID accession number EPI_ISL_417443) were previously reported. ${ }^{13}$ SARS-CoV (GZ50, GenBank accession number AY304495) was an archived clinical isolate. These viruses were propagated in Vero E6 cells and titrated with plaque assay as we described previously. ${ }^{7}$ The differentiated human airway organoids were sheared mechanically and incubated with the cultured virus for 2 hours at $37^{\circ} \mathrm{C}$. The inoculated organoids were re-embedded into Matrigel and then maintained in Advanced DMEM/F-12 (Gibco) supplemented with 1\% HEPES, 1\% GlutaMAX and 1\% Penicillin/Streptomycin. To assess replication kinetics, SARS-CoV-2 was inoculated in organoids at a MOI of 1 . At the indicated hours after inoculation, cell-free culture media were harvested and applied to RNA extraction using the MiniBEST Viral RNA/DNA Extraction Kit (Takara) and detection of viral loads (viral gene copy number of RdRp and NP gene) by one-step RT-qPCR assay, and viral titration by TCID $_{50}$ assay as described previously. ${ }^{19}$ 
To assess cellular responses upon infection, SARS-CoV-2 and SARS-CoV were inoculated in organoids at a MOI of 2. The infected or mock-infected human airway organoids were harvested at $48 \mathrm{hpi}$, applied to RNA extraction, followed by reverse transcription and qPCR assay to measure mRNA expression levels of IFNs (Supplementary Table). All experiments with live viruses were conducted in biosafety level 3 laboratories after approval by the Faculty of Medicine, The University of Hong Kong.

Transmission electron microscopy.

SARS-CoV-2-infected (5 MOI) or mock-infected human airway organoids were embedded in resin after sequential fixation in $2.5 \%$ glutaraldehyde and $1 \%$ osmium. The ultrathin sections were stained with uranyl acetate and examined under a FEI Tecnai G2 20 S-TWIN Scanning Transmission Electron Microscope.

\section{Immunofluorescence staining.}

The virus-inoculated or mock-infected human airway organoids, after fixation with 4\% PFA, permeabilization with $0.5 \%$ Triton $\mathrm{X}-100$, blocking with protein block (Dako), were applied to immunofluorescence staining using an in-house-made antibody against SARS-CoV-2 NP raised in guinea pig and a secondary antibody, Goat anti-Guinea Pig IgG Alexa Fluor 488 (A-11073, Invitrogen), to identify SARS-CoV-2 infected cells. Co-staining of NP and FOXJ1 or P63 was performed using an anti-FOXJ1 (HPA005714, Sigma-Aldrich) and anti-P63 (ab124762, abcam) respectively. ACE2 protein in the human airway organoids was labelled with an anti-ACE2 (ab15348, abcam) and Goat-anti-Rabbit secondary antibody (A-11034, Invitrogen). Nuclei and actin filaments were counterstained with DAPI (Thermo Fisher Scientific) and Phalloidin-647 (Sigma-Aldrich), respectively. The organoids were then whole mounted on glass slide with ProLong ${ }^{\mathrm{TM}}$ Glass Antifade Mountant (Invitrogen). The confocal images were acquired using a Carl Zeiss LSM 800 confocal microscope.

Flow cytometry analysis.

After an MOI of 10 inoculation, virus-inoculated or mock-infected human airway organoids were dissociated into single cells with 10mM EDTA (Invitrogen), fixed with 4\% PFA, permeabilized with $0 \cdot 1 \%$ Triton $\mathrm{X}-100$, and then applied to immunostaining using anti-dsRNA (10010500, Scicons) and Goat anti-Mouse secondary antibody (A-11001, Invitrogen) to determine the percentage of virus-infected cells. A BD FACSCantoII Analyzer was used to analyze the samples. Statistical analysis. 
Statistical analysis was conducted using GraphPad Prism 7·0. Student's t-test was used to determine statistical significance. Numbers of replicates are indicated in the figure legends. ${ }^{*} \mathrm{p} \leq$ $0 \cdot 05, * * \mathrm{p} \leq 0 \cdot 01, * * * \mathrm{p} \leq 0 \cdot 001$.

\section{Materials and Methods}

Establishment, maintenance and differentiation of human airway organoids.

After ethics approval by the Institutional Review Board of the University of Hong Kong/Hospital Authority Hong Kong West Cluster (UW13-364), three lines of human lung organoids were previously established using the human lung tissues from patients who underwent surgical resection. ${ }^{7}$ Briefly, human lung organoids are maintained in the expansion medium and passaged every 2-3 weeks. To induce differentiation into airway organoids, undifferentiated lung organoids are transferred into differentiation medium and incubated for 12-14 days. The undifferentiated lung organoids and differentiated airway organoids were harvested and applied to RNA extraction using MiniBEST Universal RNA Extraction kit (Takara), followed by reverse transcription using Transcriptor First Strand cDNA Synthesis Kit (Roche) and oligo(dT) primer. The resultant cDNAs were used to measure mRNA expression levels of host genes using the LightCycler 480 SYBR Green I Master Mix (Roche). Photomicrographs of the organoids were acquired using Nikon Eclipse TS100 Inverted Routine Microscope. Unless stated otherwise, all examinations and infections were conducted in the differentiated human airway organoids.

Virus infection and detection.

SARS-CoV-2 (GenBank accession number MT230908) and SARS-CoV-2 with deletion mutant (GISAID accession number EPI_ISL_417443) were previously reported. ${ }^{13}$ SARS-CoV (GZ50, GenBank accession number AY304495) was an archived clinical isolate. These viruses were propagated in Vero E6 cells and titrated with plaque assay as we described previously. ${ }^{7}$ The differentiated human airway organoids were sheared mechanically and incubated with the cultured virus for 2 hours at $37^{\circ} \mathrm{C}$. The inoculated organoids were re-embedded into Matrigel and then maintained in Advanced DMEM/F-12 (Gibco) supplemented with 1\% HEPES, 1\% GlutaMAX and $1 \%$ Penicillin/Streptomycin. To assess replication kinetics, SARS-CoV-2 was inoculated in organoids at a MOI of 1 . At the indicated hours after inoculation, cell-free culture media were harvested and applied to RNA extraction using the MiniBEST Viral RNA/DNA Extraction Kit 
506 (Takara) and detection of viral loads (viral gene copy number of RdRp and NP gene) by one-step

507 RT-qPCR assay, and viral titration by TCID 50 assay as described previously. ${ }^{19}$

508 To assess cellular responses upon infection, SARS-CoV-2 and SARS-CoV were inoculated in 509 organoids at a MOI of 2. The infected or mock-infected human airway organoids were harvested 510 at $48 \mathrm{hpi}$, applied to RNA extraction, followed by reverse transcription and qPCR assay to measure 511 mRNA expression levels of IFNs (Supplementary Table). All experiments with live viruses were 512 conducted in biosafety level 3 laboratories after approval by the Faculty of Medicine, The 513 University of Hong Kong.

514 Transmission electron microscopy.

515 SARS-CoV-2-infected (5 MOI) or mock-infected human airway organoids were embedded in 516 resin after sequential fixation in $2.5 \%$ glutaraldehyde and $1 \%$ osmium. The ultrathin sections were 517 stained with uranyl acetate and examined under a FEI Tecnai G2 20 S-TWIN Scanning 518 Transmission Electron Microscope.

519 Immunofluorescence staining.

520 The virus-inoculated or mock-infected human airway organoids, after fixation with 4\% PFA, 521 permeabilization with $0 \cdot 5 \%$ Triton X-100, blocking with protein block (Dako), were applied to 522 immunofluorescence staining using an in-house-made antibody against SARS-CoV-2 NP raised 523 in guinea pig and a secondary antibody, Goat anti-Guinea Pig IgG Alexa Fluor 488 (A-11073, 524 Invitrogen), to identify SARS-CoV-2 infected cells. Co-staining of NP and FOXJ1 or P63 was 525 performed using an anti-FOXJ1 (HPA005714, Sigma-Aldrich) and anti-P63 (ab124762, abcam) 526 respectively. ACE2 protein in the human airway organoids was labelled with an anti-ACE2 527 (ab15348, abcam) and Goat-anti-Rabbit secondary antibody (A-11034, Invitrogen). Nuclei and 528 actin filaments were counterstained with DAPI (Thermo Fisher Scientific) and Phalloidin-647 529 (Sigma-Aldrich), respectively. The organoids were then whole mounted on glass slide with 530 ProLong ${ }^{\text {TM }}$ Glass Antifade Mountant (Invitrogen). The confocal images were acquired using a 531 Carl Zeiss LSM 800 confocal microscope.

532 Flow cytometry analysis.

533 After an MOI of 10 inoculation, virus-inoculated or mock-infected human airway organoids were 534 dissociated into single cells with 10mM EDTA (Invitrogen), fixed with 4\% PFA, permeabilized 535 with $0 \cdot 1 \%$ Triton $\mathrm{X}-100$, and then applied to immunostaining using anti-dsRNA (10010500, 
536 Scicons) and Goat anti-Mouse secondary antibody (A-11001, Invitrogen) to determine the 537 percentage of virus-infected cells. A BD FACSCantoII Analyzer was used to analyze the samples. 538 Statistical analysis.

539 Statistical analysis was conducted using GraphPad Prism 7·0. Student's t-test was used to 540 determine statistical significance. Numbers of replicates are indicated in the figure legends. ${ }^{*} \mathrm{p} \leq$ $541 \quad 0 \cdot 05, * * \mathrm{p} \leq 0 \cdot 01, * * * \mathrm{p} \leq 0 \cdot 001$. 


\section{Figures}

Fig. 1
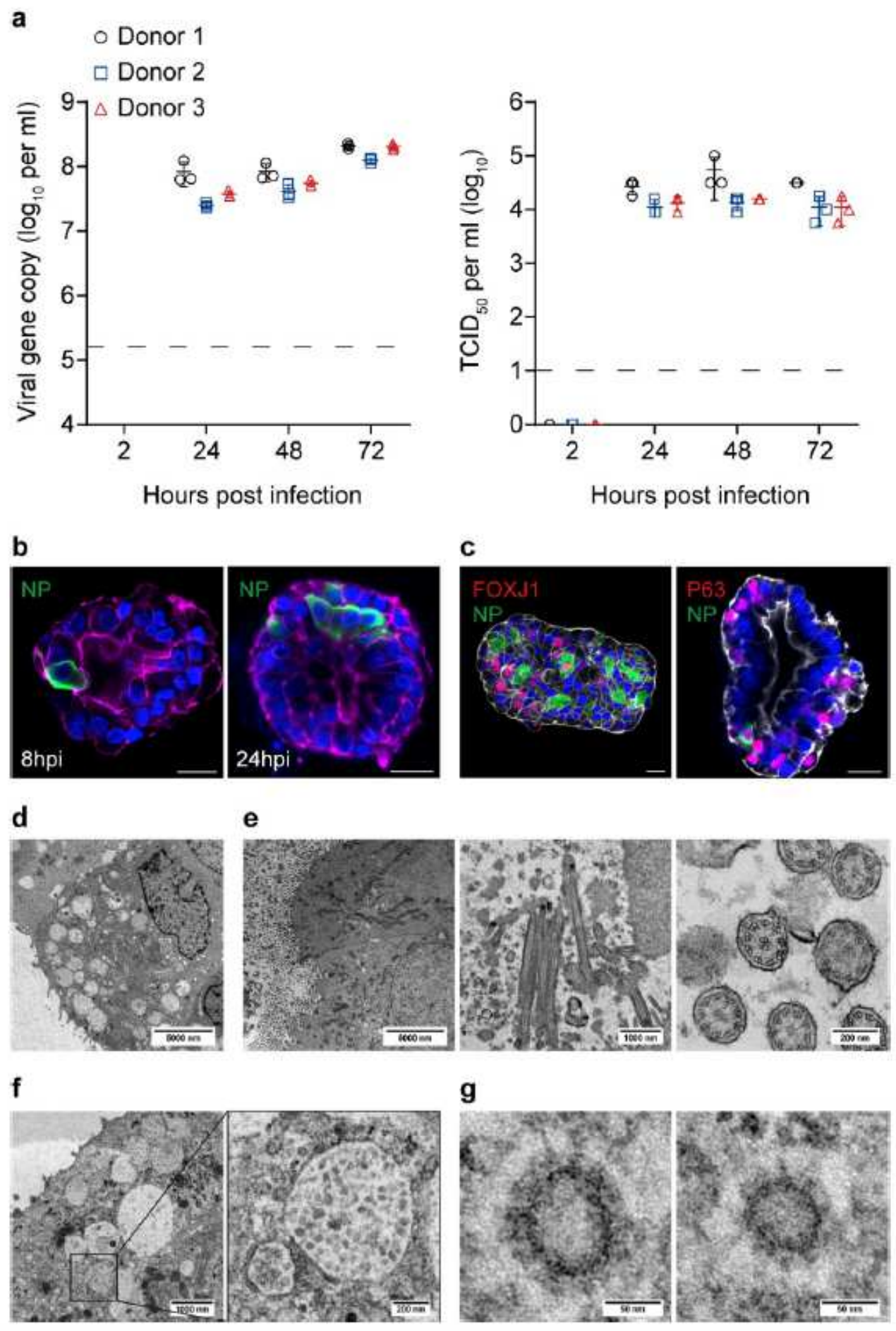

\section{Figure 1}

Active replication of SARS-CoV-2 in human airway organoids (HAO). (a) Culture media were harvested from three lines of SARS-CoV-2 infected HAO at the indicated hours post infection and applied to viral load detection and viral titration by TCID50 assay. Data represent mean and SD of a representative 
experiment, $\mathrm{n}=3$. Independent experiments were performed three times in triplicate. Dashed line indicates detection limit. (b) SARS-CoV-2-infected HAOs were fixed at 8 or $24 \mathrm{hpi}$ and immuno-stained to identify viral NP (green) positive cells. Nuclei and actin filaments were counterstained with DAPI (blue) and Phalloidin-647 (purple) respectively. Scale bar, $20 \mu \mathrm{m}$. (c) After fixation at $24 \mathrm{hpi}$, SARS-CoV-2-infected HAOs were co-stained with a-NP (green) and a-FOXJ1 (red, left) or a-P63 (red, right) to examine cell tropism. Nuclei and actin filaments were counterstained with DAPI (blue) and Phalloidin-647 (white) respectively. Scale bar, $20 \mu \mathrm{m}$. (d) Transmission electron microscopy shows ultrastructural morphology of a goblet cell in HAO. (e) Images of transmission electron microscopy illustrate ultrastructural morphology of ciliated cells (left) in $\mathrm{HAO}$, including the longitudinal section (middle) and cross-section (right) of cilia with microtubules of 9+2 arrangement. (f)Transmission electron microscopy shows the presence of virion particles in a secretory vesicle in SARS-CoV-2-infected cells in HAO; the boxed area is amplified on the right. (g) Images of transmission electron microscopy show the detailed morphology of virion particles in cytoplasm of SARS-CoV-2-infected cells in HAO.
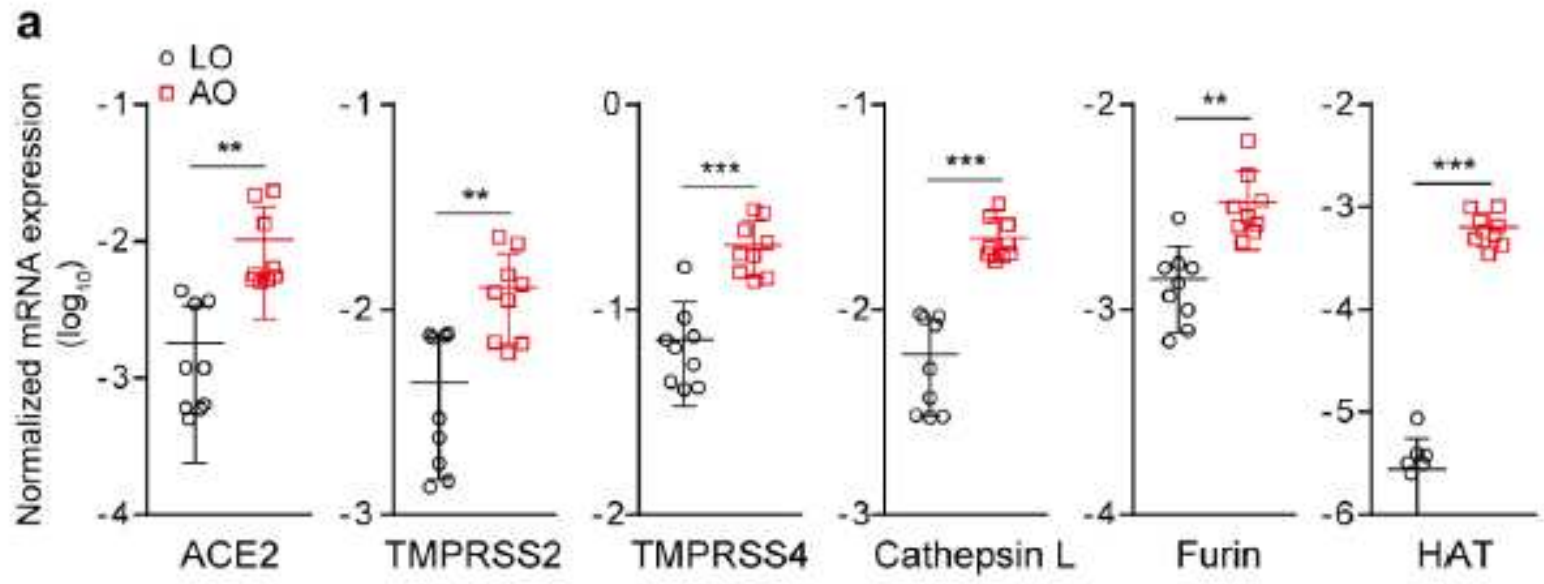

b
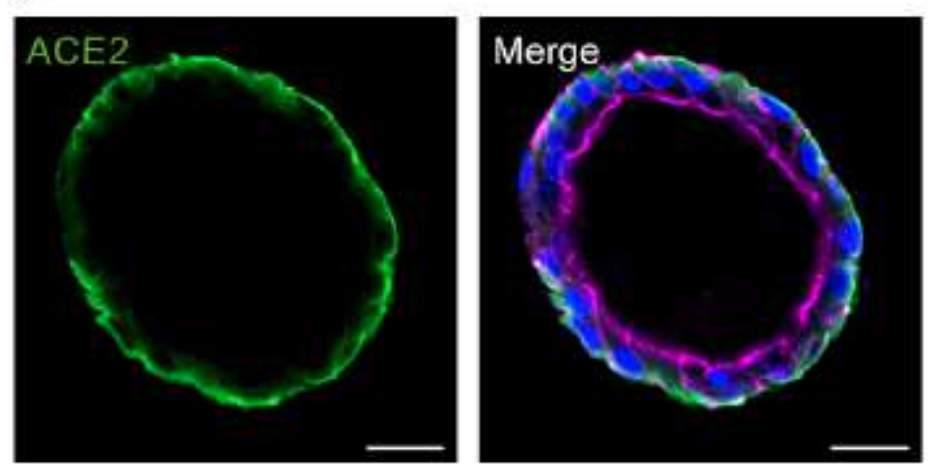

\section{Figure 2}

Expression of pro-viral host factors in human airway organoids. (a) Three lines of undifferentiated human lung organoids (LO) and the differentiated human airway organoids $(A O)$ derived from the former were applied to RT-qPCR assay to detect transcriptional levels of ACE2, TMPRSS2, TMPRSS4, Cathepsin L, Furin and HAT. Data represent mean and SD of three independent experiments, $n=9$. Two-tailed Student's 
$t$ test. (b) Differentiated human airway organoids were applied to immunofluorescence staining to label human ACE2 protein (green). Nuclei and actin filaments were counterstained with DAPI (blue) and Phalloidin-647 (purple) respectively. Scale bar, $20 \mu \mathrm{m}$.
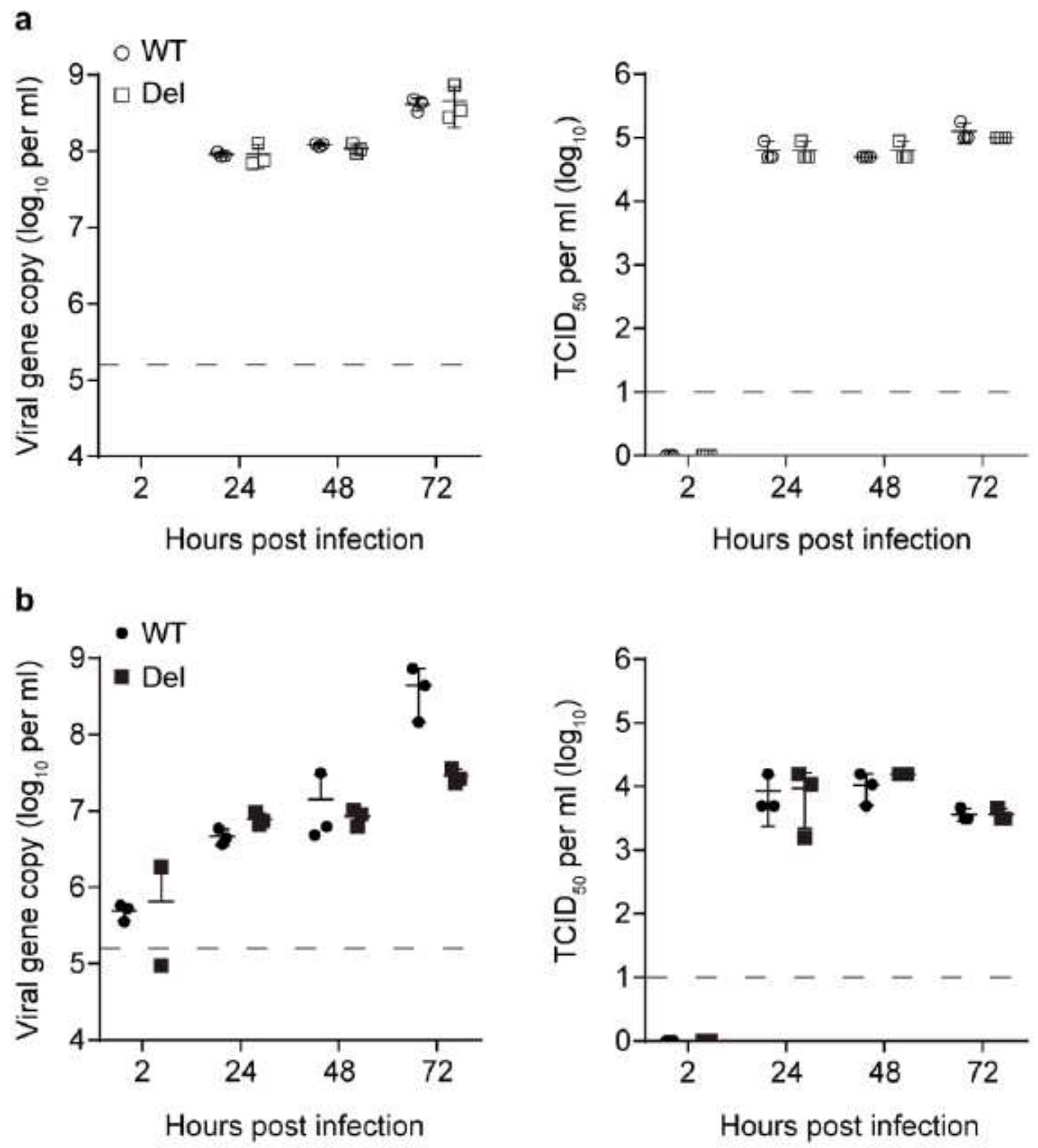

Figure 3

Comparison of wildtype and deletion mutant SARS-CoV-2 in human airway and intestinal organoids. (a) At the indicated hour post infection of wildtype (WT) or deletion mutant (Del) SARS-CoV-2 in human airway organoids, culture media were harvested and applied to viral load detection and viral titration by TCID50 assay. Data represent mean and SD of a representative experiment, $n=3$. Independent experiments were performed three times in duplicate or triplicate. Dashed line indicates detection limit. (b) Culture media were harvested from SARS-CoV-2 wildtype (WT) or deletion mutant (Del) infected human 
intestinal organoids and applied to viral load detection and viral titration by TCID50 assay. Data represent mean and SD of a representative experiment, $n=3$. Independent experiments were performed three times in triplicate. Dashed line indicates detection limit.
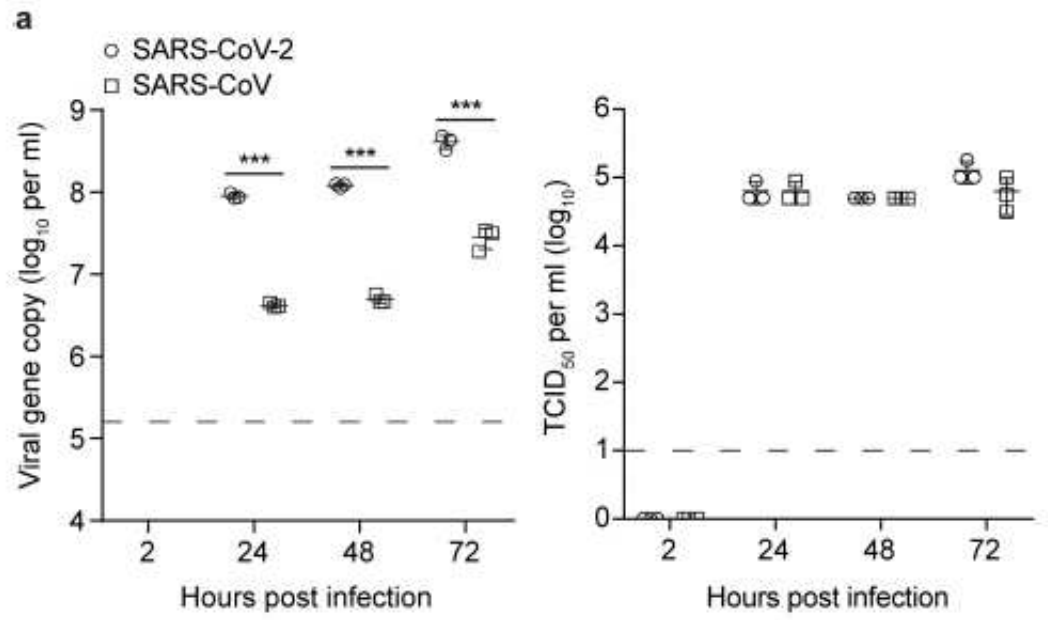

b
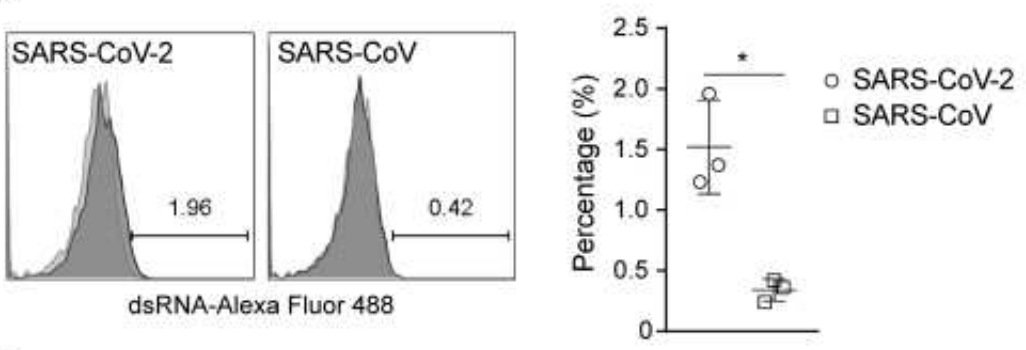

C
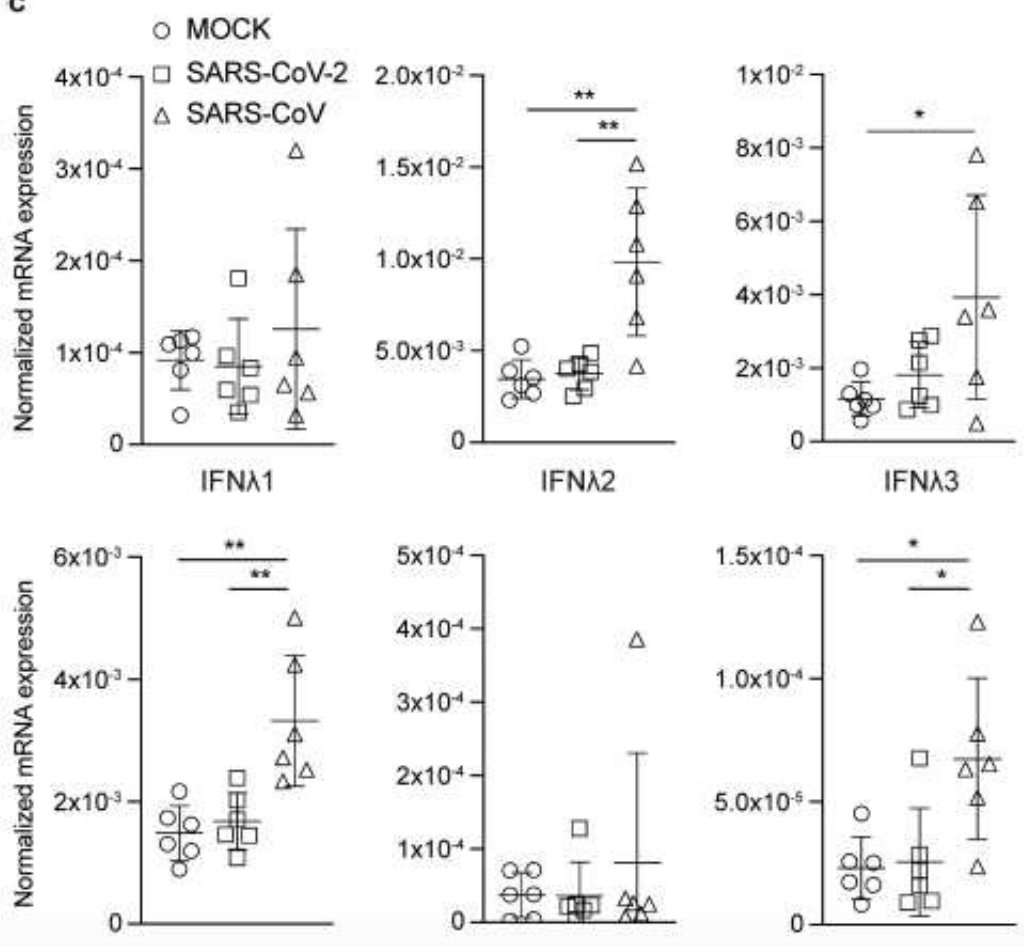

IFNa

IFN $\beta$

IFNy

\section{Figure 4}

Comparison of viral growth of SARS-CoV-2 and SARS-CoV and IFN response in human airway organoids. (a) At the indicated hour post infection of SARS-CoV-2 or SARS-CoV, culture media were harvested from 
the human airway organoids and applied to viral load detection and viral titration by TCID50 assay. Data represent mean and SD of a representative experiment, $n=3$. Independent experiments were performed three times in triplicate. Dashed line indicates detection limit. Two-tailed Student's $t$ test. (b) Representative histograms show the percentage of virus positive cells in human airway organoids infected by SARS-CoV-2 or SARS-CoV at 24 hours post infection. Data on the right panel represent mean and SD of a representative experiment, $n=3$. Two-tailed Student's t test. (c) Induction of IFNs in human airway organoids at 48 hours post infection. Results show GAPDH normalized expression level in the infected human airway organoids and the mock-infected organoids. Data represent mean and SD of two independent experiments, $n=6$. Two-tailed Student's $t$ test.

\section{Supplementary Files}

This is a list of supplementary files associated with this preprint. Click to download.

- Supplementaryinformation.docx 